\title{
Hormone replacement therapy and Parkinson's disease risk in women: a meta-analysis of 14 observational studies
}

This article was published in the following Dove Press journal:

Neuropsychiatric Disease and Treatment

31 December 2014

Number of times this article has been viewed

\section{Peifu Wang \\ Jilai Li \\ Shi Qiu \\ Honfeng Wen \\ Jichen $\mathrm{Du}$}

Department of Neurology, Aerospace Center Hospital, Peking University Aerospace Clinical College, Beijing, People's Republic of China
Correspondence: Jichen Du Department of Neurology, Aerospace Center Hospital, Peking University Aerospace Clinical College, 15 Yuquan Road, Beijing I00049, People's Republic of China

Email jichen_du@I63.com
Background and purpose: Published data on the relationship of hormone replacement therapy (HRT) with Parkinson's disease (PD) were inconclusive. Thus, a systematic meta-analysis of observational studies was performed to clarify this topic.

Methods: The databases of PubMed and EMBASE were searched for case-control or cohort studies published up till June 2, 2014. Meta-analysis of the relative risks (RRs) with $95 \%$ confidence intervals (CIs) was estimated using random-effects models.

Results: A final total of ten case-control and four cohort studies were included in our metaanalysis. The overall combined RR of PD for ever users versus never users of HRT was 1.00 (95\% CI: $0.84-1.20)$. Limited to those subjects who only use estrogen, a similar trend was detected (RR: $0.95,95 \%$ CI: 0.69-1.30). In the subgroup analysis by study design, no significant association was observed in case-control studies (RR: 0.79, 95\% CI: 0.62-1.02), whereas a positive association was found in cohort studies (RR: 1.24, 95\% CI: 1.10-1.40). In further analysis according to study quality, an inverse association was found in the low-quality group (RR: $0.58,95 \%$ CI: $0.40-0.82$ ), whereas a positive association was found in the high-quality group (RR: $1.16,95 \% \mathrm{CI}: 1.02-1.31$ ).

Conclusion: In summary, our results of meta-analysis do not support a protective role of HRT in female PD development.

Keywords: Parkinson's disease, hormone replacement therapy, hormone meta-analysis

\section{Introduction}

Parkinson's disease (PD) is the second most frequent type of neurodegenerative disorder. ${ }^{1}$ The cardinal symptoms of PD are static tremors, rigidity, bradykinesia, and impairment of postural reflexes. ${ }^{2}$ Despite decades of research, the cause of PD is poorly understood. To date, age is the only proven risk factor for PD. ${ }^{3,4}$ Several other risk factors such as gene polymorphism, tobacco use, alcohol and caffeine consumption, pesticides, and history of head trauma, hypertension, and diabetes mellitus have been widely investigated, but the impact of most of these factors on the risk of developing PD remains uncertain. ${ }^{4,5}$

$\mathrm{PD}$ is more common in men than in women, with a male-to-female ratio of ageadjusted incidence rates of $1.5,{ }^{6}$ and experimental studies have shown a neuroprotective effect of estrogen on the nigrostriatal dopaminergic system. ${ }^{7}$ Based on the sex difference and experimental evidence, the role of female hormones in the etiology of PD has been the subject of speculation for many years. Epidemiologic studies evaluating the relationship between the risk of PD and hormone replacement therapy (HRT) in women have yielded inconsistent results. ${ }^{8-21}$ This discrepancy in the results may be 
related to insufficient statistical power of individual studies. Thus, a meta-analysis of published case-control and cohort studies was performed to derive the most precise estimation of association between risk of PD and HRT.

\section{Materials and methods Search strategy}

Studies on the association between risk of PD and HRT published before June 2, 2014 were identified from PubMed and EMBASE databases. No language limitation was imposed during our literature search. The search string was as follows: ("hormone" OR "exogenous hormones" OR "exogenous hormones use" OR "hormone replacement therapy" OR "menopausal hormone therapy" OR "estrogen replacement therapy" OR "menopausal hormone use") AND ("Parkinson's disease" OR "shaking palsy" OR "Parkinsonism" OR "paralysis agitans"). Furthermore, reference lists of relevant articles and reviews were also screened for additional studies.

\section{Selection criteria}

The following criteria were adopted to identify eligible studies: 1) a case-control or cohort study evaluating the relationship between $\mathrm{PD}$ risk and $\mathrm{HRT}$; 2) reporting the estimates of the relative risk ( $\mathrm{RR})$ /odds ratio/hazard ratio with corresponding 95\% confidence intervals (CIs) or data sufficient to calculate them; 3) when more than one report was published on the same study population, we considered only the most informative one in our analysis; 4) we excluded review, meta-analysis, conference abstract, and cross-sectional studies.

\section{Data extraction and assessment of methodological quality}

Data extraction and study quality assessment were independently conducted by two authors. The data extracted from each article included the first author's surname, publication year, area where the study was performed, study design, sample size, method of exposure assessment, type of hormone use, covariates for adjustment, and the adjusted risk estimates with corresponding $95 \%$ CIs. Assessment of research quality was performed according to the criteria of the NewcastleOttawa Scale (NOS), which was proposed by Wells for meta-analysis of observational studies. ${ }^{22}$ Detailed grading standards consist of: selection (maximum score $=4 *$ ), comparability (maximum score $=2 *$ ), and exposure or outcome (maximum score $=3^{*}$ ). In our meta-analysis, a study with more than six points was considered to be of relatively higher quality.

\section{Statistical analysis}

All statistical analyses were conducted with STATA software (v12.0; College Station, TX, USA). The pooled RRs with corresponding $95 \%$ CIs were calculated with a random-effects model (DerSimonian-Laird method). ${ }^{23}$ Statistical heterogeneity was assessed using the $Q$ statistic (defining significant heterogeneity as a $P$-value of $<0.10$ ) and $I^{2}$ statistic (defining significant heterogeneity as $\left.P^{2}>50 \%\right){ }^{24,25}$ Subgroup analyses were performed according to study design (cohort vs case-control studies), type of controls for the case-control studies (population-based vs hospital-based controls studies), geographic region (North America vs Europe), study quality (high vs low), type of HRT (estrogen vs progesterone), and adjustment for age (yes vs no). We performed sensitivity analysis by excluding one study at a time to evaluate the robustness of the overall results. Publication bias was evaluated using Egger's linear regression test. ${ }^{26}$

As the most frequent definition of exposure among the studies was "ever versus never use of hormone replacement therapy", this was chosen to be the focus of the main analysis. Moreover, short-term use of HRT was defined as a duration of $<5$ years, and long-term use as a duration of $\geq 5$ years. When more than one exposure in a study category fell into the same level, we pooled the corresponding estimates using Hamling's method. ${ }^{27}$ This method takes into account the correlation between estimates.

\section{Results}

\section{Search results and study characteristics}

Figure 1 provides the flowchart for the selection of articles. We identified 2,193 publications from the database search. Based on our inclusion criteria, a total of 14 studies published between 1998 and 2014 were included. ${ }^{8-21}$ Table 1 presents the main characteristic of the included studies. There were ten case-control and four cohort studies. Seven studies were performed in the US, four in Italy, two in Denmark, and one in Belgium. Regarding the type of hormone use, seven studies focused on the use of estrogen, five involved total hormone use, and two studies provided data on special types of hormones (ie, estrogen and/or progesterone). Data on hormone use were ascertained by a self-reported questionnaire, telephone interview, face-to-face interview, or reviewing medical records. The type and number of potential confounders varied between studies: age, race, smoking, coffee/caffeine, education, positive familial history, age at menopause, type of menopause, alcohol, oral contraceptive, parity, respondent type, pesticide use, degree of urbanization, family PD history, and age at first symptom. 


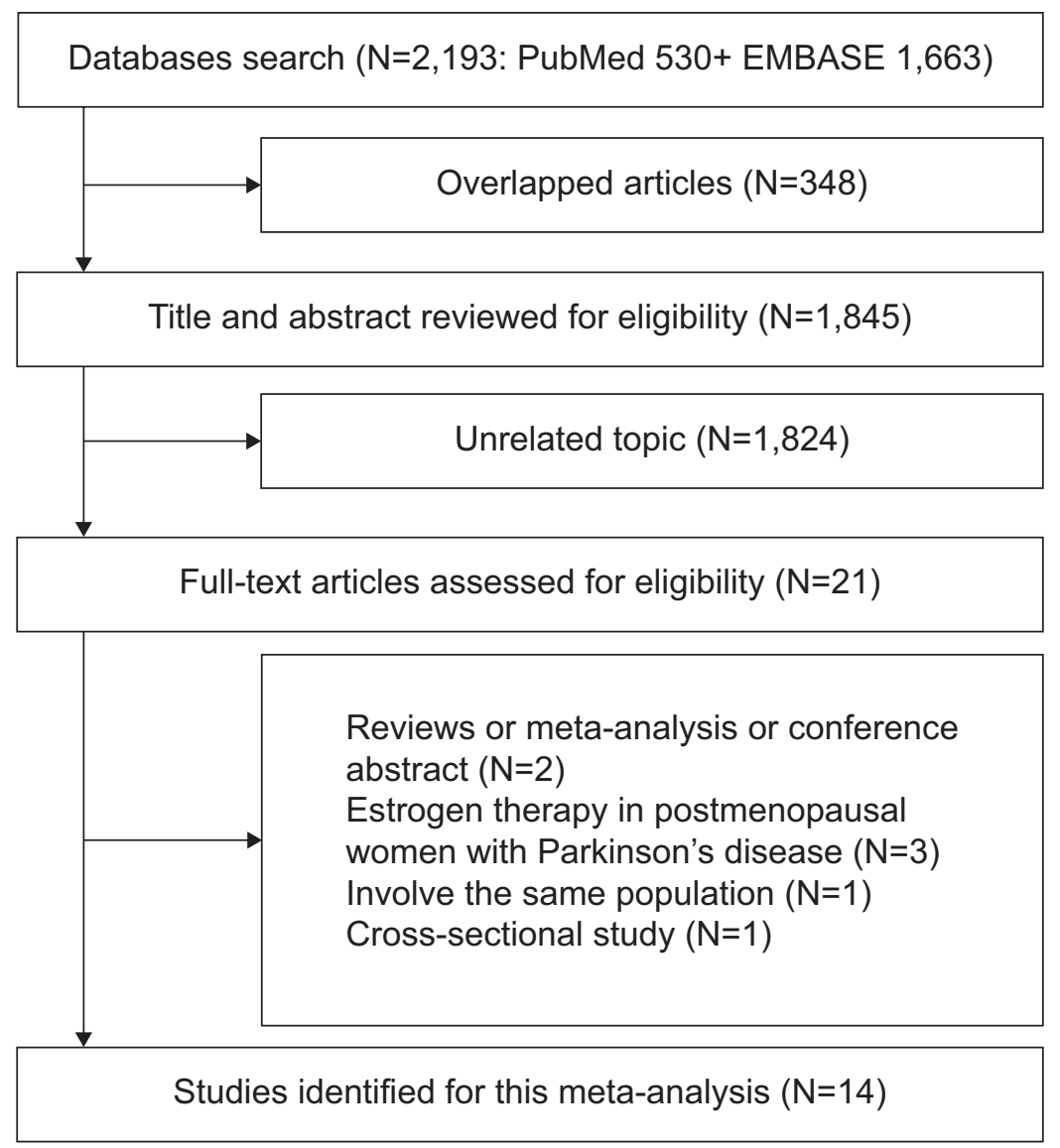

Figure I Flow diagram of literature search and selection.

\section{Synthesis of results for hormone replacement therapy and risk of Parkinson's disease}

Figure 2 shows the forest plots for ever users versus never users of HRT. The pooled RR for case-control, cohort studies, and all studies were 0.79 (95\% CI: $0.62-1.02, P_{Q}=0.189$, $\left.I^{2}=27.8 \%\right), 1.24$ (95\% CI: $\left.1.10-1.41, P_{Q}=0.758, I^{2}=0.0 \%\right)$, and 1.00 (95\% CI: $\left.0.84-1.20, P_{Q}=0.016, I^{2}=50.3 \%\right)$, respectively. In further analysis according to type of controls for the case-control studies, no significant association was observed in population-based case-control studies or hospital-based case-control studies (Table 2).

In the subgroup analysis by geographic region, the pooled risk estimates were 1.12 (95\% CI: 0.92-1.36, $P_{Q}=0.074$, $\left.I^{2}=47.9 \%\right)$ for North America and 0.86 (95\% CI: 0.63-1.16, $P_{Q}=0.162, I^{2}=34.8 \%$ ) for European countries.

In the subgroup analysis by study quality, an inverse association was found in the low-quality group (RR: 1.16, 95\% CI: $1.02-1.31, P_{Q}=0.309, P^{2}=14.6 \%$ ), whereas a positive association was found in the high-quality group (RR: 0.58 , 95\% CI: $0.40-0.82, P_{Q}=0.633, I^{2}=0.0 \%$ ).
In the subgroup analysis by adjustment for age, no significant correlation was observed neither in the adjusted group (RR: $1.05,95 \%$ CI: $0.88-1.26, P_{Q}=0.050, I^{2}=46.8 \%$ ) nor in the unadjusted group (RR: $0.87,95 \% \mathrm{CI}$ : $0.49-1.53$, $P_{Q}=0.057, I^{2}=60.1 \%$ ).

In the subgroup analysis by type of female hormone use, the cumulative estimated risks associated with ever use of estrogen replacement therapy (ERT) was 1.05 (95\% CI: $0.79-$ $1.40, P_{Q}=0.021, I^{2}=54.1 \%$ ). Among the included studies, only one study assessed the association between progesterone use and PD risk (RR: 3.41, 95\% CI: 1.23-9.47).

Four studies provided data on the duration of HRT use and PD risk. ${ }^{13,17,19,21}$ The pooled RRs were 1.22 (95\% CI: $1.03-$ 1.44, $\left.P_{Q}=0.954, I^{2}=0.0 \%\right)$ for short-term use and $1.15(95 \%$ CI: $0.97-1.34, P_{Q}=0.259, I^{2}=25.4 \%$ ) for long-term use.

\section{Sensitivity analysis and publication bias}

Sensitivity analyses were performed to evaluate the effect of one single study on the overall estimate by sequentially excluding each study at one time. The sensitivity analyses suggested that the overall result was robust (Figure 3). 


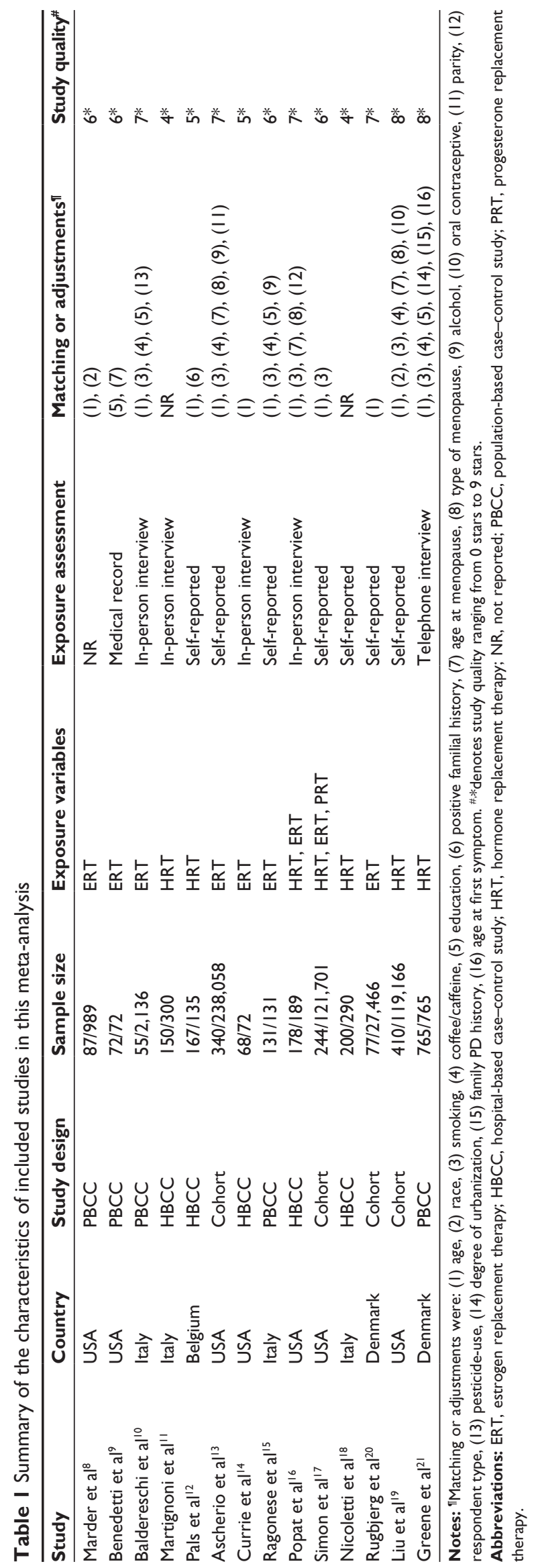

A publication bias was identified using Egger's linear regression test $(P=0.013)$. Then, the trim-and-fill method, which estimates the number of potential missing studies resulting from publication bias, was also implemented. ${ }^{28}$ This method identified any possible missing studies; the adjustmentestimated RR with corresponding 95\% CI was not changed, indicating that our result was stable.

\section{Discussion}

In this meta-analysis of 14 case-control and cohort studies involving more than 2,900 cases and 511,000 non-case subjects, HRT does not appear to be associated with PD risk. The results from this meta-analysis are similar to those conducted by Noyce et al. ${ }^{5}$ The previous meta-analysis of nine observational studies found a significant inverse association between HRT and PD risk in case-control studies, but a significant positive association in cohort studies. The main advantages of the present meta-analysis are the inclusion of a large number of subjects, addressing the variability between studies (ie, type of controls for the case-control studies, geographic region, study quality, type of HRT, adjustment factor, and method of exposure assessment), and performing dose-response analysis of duration. Therefore, our results provided a more precise and valid assessment of the association between HRT and PD risk.

In the subgroup analysis according to geographic area, no significant association was observed in the North America or Europe studies. When stratified by quality of the included studies, an inverse association was observed in the lowquality group. However, a positive association emerged for the high-quality group. This discrepancy may be related to the study design. In the low-quality group, the included studies were hospital-based case-control studies. The likelihood of recall and selective biases may be greatly increased. Additionally, in further analyses according to adjustment for age, no significant correlation was observed in the adjusted group or in the unadjusted group. Thus, findings from the high-quality group may be distorted by age, a proven risk factor for PD.

The correlation between specific types of HRT (ie, estrogen and/or progesterone) and PD risk was explored in nine studies. ${ }^{8-10,13-17,20}$ The risk estimates were not significant, except for two, from the Cancer Prevention Study $\mathrm{II}^{13}$ and the Kaiser Permanente Medical Care Program (KPMCP) of Northern California. ${ }^{16}$ Ascherio et al found ERT was correlated with a higher risk of death from PD. ${ }^{13}$ In KPMCP, estrogen use was associated with a 2.6-fold increased risk of PD. ${ }^{16}$ In our meta-analysis, we found no evidence of a 


\begin{tabular}{|c|c|c|}
\hline Study ID & $\mathrm{RR}(95 \% \mathrm{Cl})$ & $\%$ weight \\
\hline \multicolumn{3}{|l|}{ Case-control studies } \\
\hline Marder et $\mathrm{al}^{8}$ & $1.02(0.56-1.80)$ & 6.15 \\
\hline Benedetti et $\mathrm{al}^{9}$ & $0.47(0.12-1.85)$ & 1.53 \\
\hline Baldereschi et al ${ }^{10}$ & $0.55(0.17-1.80)$ & 1.99 \\
\hline Martignoni et $\mathrm{al}^{11}$ & $0.62(0.40-0.98)$ & 8.51 \\
\hline Pals et al ${ }^{12}$ & $0.53(0.13-2.11)$ & 1.47 \\
\hline Currie et $\mathrm{al}^{14}$ & $0.40(0.19-0.84)$ & 4.33 \\
\hline Ragonese et al ${ }^{15}$ & $0.45(0.13-1.50)$ & 1.87 \\
\hline Popat et al ${ }^{16}$ & $1.30(0.80-2.10)$ & 7.82 \\
\hline Nicoletti et al ${ }^{18}$ & $0.99(0.27-3.57)$ & 1.70 \\
\hline Greene et $\mathrm{al}^{21}$ & $0.94(0.71-1.24)$ & 12.84 \\
\hline Subtotal $\left(P^{2}=27.8 \%, P=0.189\right)$ & $0.79(0.62-1.02)$ & 48.21 \\
\hline \multicolumn{3}{|l|}{ Cohort studies } \\
\hline Ascherio et al ${ }^{13}$ & $1.33(1.07-1.67)$ & 14.53 \\
\hline Simon et al $\left.\right|^{17}$ & $1.14(0.87-1.48)$ & 13.23 \\
\hline Liu et $\mathrm{al}^{19}$ & $1.21(1.00-1.46)$ & 15.53 \\
\hline Rugbjerg et $a^{20}$ & $1.42(0.91-2.23)$ & 8.51 \\
\hline Subtotal $\left(P^{2}=0.0 \%, P=0.758\right)$ & $1.24(1.10-1.41)$ & 51.79 \\
\hline Overall $(R=50.3 \%, P=0.016)$ & $1.00(0.84-1.20)$ & 100.00 \\
\hline$T$ & $T$ & \\
\hline 0.12 & 8.33 & \\
\hline
\end{tabular}

Figure 2 Forest plots of HRT and PD risk.

Note: Weights are from random-effects analysis.

Abbreviations: $\mathrm{Cl}$, confidence interval; HRT, hormone replacement therapy; PD, Parkinson's disease; RR, relative risk.

Table 2 Summary of RR estimates between risk of Parkinson's disease and HRT in women

\begin{tabular}{|c|c|c|c|c|}
\hline \multirow[t]{2}{*}{ Group } & \multicolumn{2}{|l|}{ Meta-analysis } & \multirow[t]{2}{*}{$P_{\mathrm{Q}}$} & \multirow[t]{2}{*}{$I^{2}$} \\
\hline & Number of studies & Pooled RR (95\% CI) & & \\
\hline All studies & 14 & $1.00(0.84-1.20)$ & 0.016 & $50.3 \%$ \\
\hline \multicolumn{5}{|l|}{ Study design } \\
\hline Case-control studies & 10 & $0.79(0.62-1.02)$ & 0.189 & $27.8 \%$ \\
\hline PBCC & 5 & $0.89(0.70-1.13)$ & 0.553 & $0.0 \%$ \\
\hline $\mathrm{HBCC}$ & 5 & $0.73(0.45-1.18)$ & 0.066 & $54.7 \%$ \\
\hline Cohort studies & 4 & $1.24(1.10-1.41)$ & 0.758 & $0.0 \%$ \\
\hline \multicolumn{5}{|l|}{ Geographic region } \\
\hline North America & 7 & $1.12(0.92-1.36)$ & 0.074 & $14.6 \%$ \\
\hline Europe & 7 & $0.86(0.63-1.16)$ & 0.162 & $34.8 \%$ \\
\hline \multicolumn{5}{|l|}{ Study quality } \\
\hline High & 10 & $1.16(1.02-1.31)$ & 0.309 & $14.6 \%$ \\
\hline Low & 4 & $0.58(0.40-0.82)$ & 0.633 & $0.0 \%$ \\
\hline \multicolumn{5}{|l|}{ Adjusted for age } \\
\hline Yes & 10 & $1.05(0.88-1.26)$ & 0.050 & $46.8 \%$ \\
\hline No & 4 & $0.87(0.49-1.53)$ & 0.057 & $60.1 \%$ \\
\hline \multicolumn{5}{|l|}{ Type of HRT } \\
\hline ERT & 9 & $1.05(0.79-1.40)$ & 0.021 & $54.1 \%$ \\
\hline PRT & 1 & $3.4 I$ (I.23-9.47) & NA & NA \\
\hline
\end{tabular}

Abbreviations: $\mathrm{Cl}$, confidence interval; ERT, estrogen replacement therapy; HBCC, hospital-based case-control study; HRT, hormone replacement therapy; NA, data not available; PBCC, population-based case-control study; PRT, progesterone replacement therapy; $P, P$-value for $Q$ statistic; RR, relative risk. 


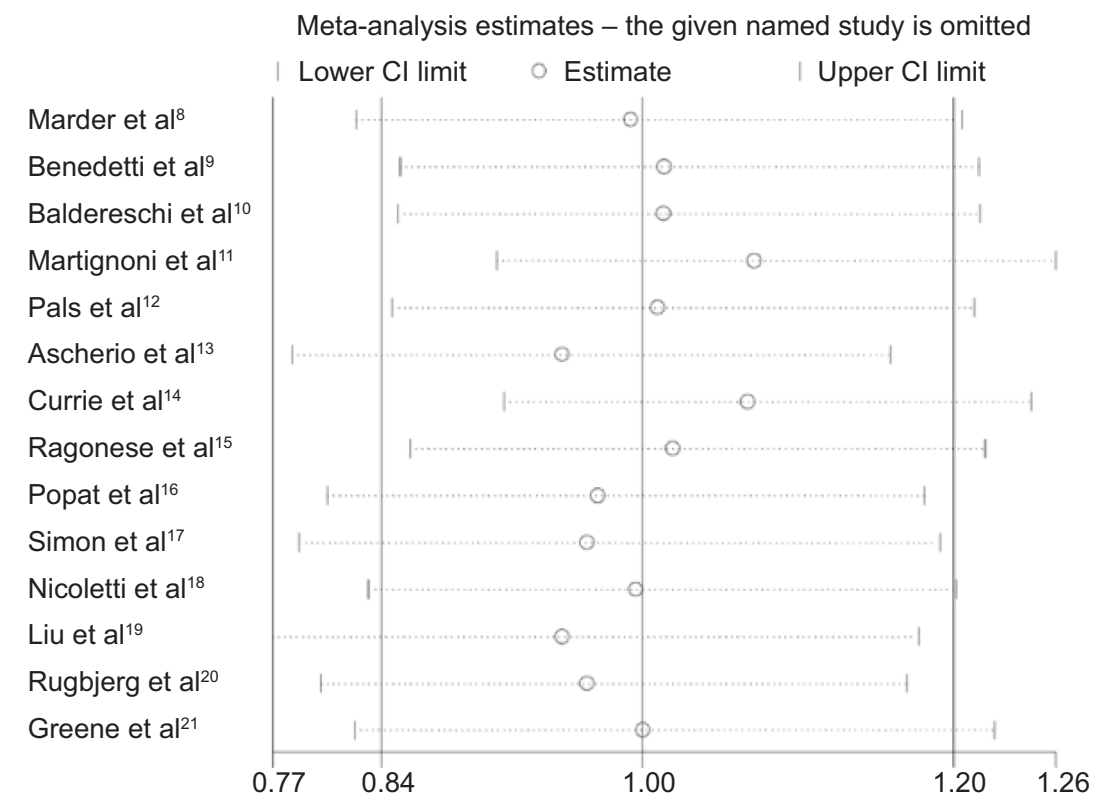

Figure 3 Sensitivity analyses for HRT and PD risk.

Abbreviations: $\mathrm{Cl}$, confidence interval; HRT, hormone replacement therapy; PD, Parkinson's disease.

meaningful correlation between ERT and PD risk. Moreover, use of progesterone replacement therapy was investigated in the Nurses' Health Study with 22 years of follow-up. ${ }^{17}$ Simon et al found the use of progestin alone was associated with a three-fold higher PD risk. ${ }^{17}$

Evaluation of a dose-risk relationship in a meta-analysis of observational studies provides evidence for a suspected cause and effect relationship between exposure and disease. Among the 14 individual studies, five studies performed a dose-response analysis of duration of HRT use. ${ }^{13,16,17,19,21}$ All of these studies showed no indication of trend for duration. However, Popat et al in KPMCP found the direction and strength of the relationship between HRT and PD risk lay on the type of menopause. ${ }^{16}$ PD risk increased with longer duration of estrogen therapy in women with a history of hysterectomy compared with never users. There was an approximately two-fold increased risk of PD associated with $\leq 10$ years of estrogen therapy and three-fold increased risk of PD associated with $>10$ years of estrogen therapy ( $P$-value for linear trend $<0.05$ ). On the contrary, decreasing risk with increasing duration of HRT emerged in women with natural menopause. The significance of this finding was unclear. Therefore, the joint effects of type of menopause and HRT on PD risk needs further discussion. In addition, we performed a meta-analysis of duration of HRT use in relation to PD risk. We defined short-term use as a duration of $<5$ years, and long-term use as a duration of $\geq 5$ years. According to our criteria, only four studies were identified for our dose-risk analysis. ${ }^{13,17,19,21}$ The results demonstrated that short-term use was associated with an increased risk of PD, but the association between long-term use and PD risk was not statistically significant. Concerning limited data involving the dose-effect analysis, we cannot exclude that our finding, of an increased risk of PD in women with short-term use of HRT, is a chance finding.

In recent years, several studies have investigated the role of HRT in the improvement of motor symptoms in postmenopausal women with PD. ${ }^{29-32}$ Blanchet et al found $17 \beta$-estradiol has a slight or antiparkinsonian effect without consistently altering dyskinesias in their double-blind, placebo-controlled, twoarm crossover study of high-dose transdermal $17 \beta$-estradiol in eight postmenopausal women with mild-to-moderate PD. ${ }^{29}$ In the latter, 8-week double-blind, parallel-group, prospective study with 40 subjects, Tsang et al found a statistically significant improvement of motor function in those women receiving low-dose estrogen $(0.625 \mathrm{mg} /$ day $)$, with a mean 3.5 point improvement on the motor Unified Parkinson's Disease Rating Scale (UPDRS) score. ${ }^{30}$ However, a placebo-controlled, randomized, double-blind trial involving 12 postmenopausal female PD patients under the age of 80 years revealed estradiol had no significant dopaminergic effect, whereas progesterone appeared to have an antidopaminergic effect. ${ }^{31}$ A more recent multicenter randomized, double-blind, placebo-controlled pilot trial from the Parkinson Study Group POETRY Investigators showed a nonsignificant trend of symptomatic and functional improvement in those receiving ERT. ${ }^{32}$ Concerning the small number of participants included in each original study, it is conceivable that the conflicting results were due to the small 
sample size. Moreover, a double-masked, placebo-controlled, parallel-group, single-center trial in 30 male PD patients investigated the effect of testosterone therapy. ${ }^{33}$ Okun et al found that there was no significant improvement of the motor and nonmotor symptoms in the testosterone therapy group compared with the placebo group. ${ }^{33}$ The authors were also aware of the possibility that the observed null effect may have resulted from various limitations, including the small sample size, a strong placebo effect with intramuscular therapy, and the short follow-up period. Therefore, whether sex hormones play a role in the improvement of PD symptoms also remains for future study and more well-designed studies are warranted to clarify this issue.

Our study also had several limitations. First, as a metaanalysis of observational studies, we cannot rule out the probability that the observed null association between HRT and PD risk could be due to unmeasured or residual confounding. Second, some PD cases were identified by self-reports. It is inevitable that some cases were missed or misdiagnosed. Thus, the true association may be underestimated to a degree. Third, misclassification or measurement errors for HRT may have distorted the association, because our analyses were based on the data which were ascertained at baseline, and the changes of exposure were not addressed during the follow-up. Fourth, publication bias was observed in our meta-analysis. However, the results from the trim-and-fill method showed the overall results were robust.

In conclusion, the present meta-analysis provided little support for the hypothesis of a protective effect of HRT on $\mathrm{PD}$ risk. However, the significantly increased risk of $\mathrm{PD}$ in cohort studies remains to be clarified.

\section{Author contributions}

Peifu Wang and Jichen Du conceived and designed the experiments. Peifu Wang and Jilai Li performed the experiments. Peifu Wang, Jilai Li, Shi Qiu, and Honfeng Wen analyzed the data. Peifu Wang and Jichen Du wrote the paper. All authors critically revised the content and approved the final manuscript.

\section{Disclosure}

The authors report no conflicts of interest in this work.

\section{References}

1. Dye RV, Miller KJ, Singer EJ, Levine AJ. Hormone replacement therapy and risk for neurodegenerative diseases. Int J Alzheimers Dis. 2012;2012:258-454.

2. Calne DB, Snow BJ, Lee C. Criteria for diagnosing Parkinson's disease. Ann Neurol. 1992;32 Suppl:S125-S127.

3. Kieburtz K, Wunderle KB. Parkinson's disease: evidence for environmental risk factors. Mov Disord. 2013;28(1):8-13.
4. Alves G, Forsaa EB, Pedersen KF, Dreetz Gjerstad M, Larsen JP. Epidemiology of Parkinson's disease. J Neurol. 2008;255 Suppl 5: 18-32.

5. Noyce AJ, Bestwick JP, Silveira-Moriyama L, et al. Meta-analysis of early nonmotor features and risk factors for Parkinson disease. Ann Neurol. 2012;72(6):893-901.

6. Wooten GF, Currie LJ, Bovbjerg VE, Lee JK, Patrie J. Are men at greater risk for Parkinson's disease than women? J Neurol Neurosurg Psychiatry. 2004;75(4):637-639.

7. Brann DW, Dhandapani K, Wakade C, Mahesh VB, Khan MM. Neurotrophic and neuroprotective actions of estrogen: basic mechanisms and clinical implications. Steroids. 2007;72(5):381-405.

8. Marder K, Tang MX, Alfaro B, et al. Postmenopausal estrogen use and Parkinson's disease with and without dementia. Neurology. 1998; 50(4):1141-1143.

9. Benedetti MD, Maraganore DM, Bower JH, et al. Hysterectomy, menopause, and estrogen use preceding Parkinson's disease: an exploratory case-control study. Mov Disord. 2001;16(5):830-837.

10. Baldereschi M, Di Carlo A, Vanni P, et al. Lifestyle-related risk factors for Parkinson's disease: a population-based study. Acta Neurol Scand. 2003;108(4):239-244.

11. Martignoni E, Nappi RE, Citterio A, et al. Reproductive life milestones in women with Parkinson's disease. Funct Neurol. 2003;18(4): 211-217.

12. Pals P, Van Everbroeck B, Grubben B, et al. Case-control study of environmental risk factors for Parkinson's disease in Belgium. Eur $J$ Epidemiol. 2003;18(12):1133-1142.

13. Ascherio A, Weisskopf MG, O’Reilly EJ, et al. Coffee consumption, gender, and Parkinson's disease mortality in the cancer prevention study II cohort: the modifying effects of estrogen. Am J Epidemiol. 2004; 160(10):977-984.

14. Currie LJ, Harrison MB, Trugman JM, Bennett JP, Wooten GF. Postmenopausal estrogen use affects risk for Parkinson disease. Arch Neurol. 2004;61(6):886-888.

15. Ragonese P, D’Amelio M, Salemi G, et al. Risk of Parkinson disease in women: effect of reproductive characteristics. Neurology. 2004; 62(11):2010-2014.

16. Popat RA, Van Den Eeden SK, Tanner CM, et al. Effect of reproductive factors and postmenopausal hormone use on the risk of Parkinson disease. Neurology. 2005;65(3):383-390.

17. Simon KC, Chen H, Gao X, Schwarzschild MA, Ascherio A. Reproductive factors, exogenous estrogen use, and risk of Parkinson's disease. Mov Disord. 2009;24(9):1359-1365.

18. Nicoletti A, Nicoletti G, Arabia G, et al. Reproductive factors and Parkinson's disease: a multicenter case-control study. Mov Disord. 2011;26(14): 2563-2566.

19. Liu R, Baird D, Park Y, et al. Female reproductive factors, menopausal hormone use, and Parkinson's disease. Mov Disord. 2014;29(7): 889-896.

20. Rugbjerg K, Christensen J, Tjønneland A, Olsen JH. Exposure to estrogen and women's risk for Parkinson's disease: a prospective cohort study in Denmark. Parkinsonism Relat Disord. 2013;19(4): $457-460$.

21. Greene N, Lassen CF, Rugbjerg K, Ritz B. Reproductive factors and Parkinson's disease risk in Danish women. Eur J Neurol. 2014; 21(9):1168-1177, e68.

22. Stang A. Critical evaluation of the Newcastle-Ottawa scale for the assessment of the quality of nonrandomized studies in meta-analyses. Eur J Epidemiol. 2010;25(9):603-605.

23. DerSimonian R, Laird N. Meta-analysis in clinical trials. Control Clin Trials. 1986;7(3):177-188.

24. Higgins JP, Thompson SG. Quantifying heterogeneity in a metaanalysis. Stat Med. 2002;21(11):1539-1558.

25. Higgins JP, Thompson SG, Deeks JJ, Altman DG. Measuring inconsistency in meta-analyses. BMJ. 2003;327(7414):557-560.

26. Egger M, Davey Smith G, Schneider M, Minder C. Bias in metaanalysis detected by a simple, graphical test. BMJ. 1997;315(7109): $629-634$. 
27. Hamling J, Lee P, Weitkunat R, Ambühl M. Facilitating meta-analyses by deriving relative effect and precision estimates for alternative comparisons from a set of estimates presented by exposure level or disease category. Stat Med. 2008;27(7):954-970.

28. Duval S, Tweedie R. Trim and fill: a simple funnel-plot-based method of testing and adjusting for publication bias in meta-analysis. Biometrics. 2000;56(2):455-463.

29. Blanchet PJ, Fang J, Hyland K, Arnold LA, Mouradian MM, Chase TN. Short-term effects of high-dose 17beta-estradiol in postmenopausal PD patients: a crossover study. Neurology. 1999;53(1):91-95.

30. Tsang KL, Ho SL, Lo SK. Estrogen improves motor disability in parkinsonian postmenopausal women with motor fluctuations. Neurology. 2000;54(12):2292-2298.
31. Strijks E, Kremer JA, Horstink MW. Effects of female sex steroids on Parkinson's disease in postmenopausal women. Clin Neuropharmacol. 1999;22(2):93-97.

32. Parkinson Study Group POETRY Investigators. A randomized pilot trial of estrogen replacement therapy in post-menopausal women with Parkinson's disease. Parkinsonism Relat Disord. 2011;17(10): 757-760.

33. Okun MS, Fernandez HH, Rodriguez RL, et al. Testosterone therapy in men with Parkinson disease: results of the TEST-PD Study. Arch Neurol. 2006;63(5):729-735.

\section{Publish your work in this journal}

Neuropsychiatric Disease and Treatment is an international, peerreviewed journal of clinical therapeutics and pharmacology focusing on concise rapid reporting of clinical or pre-clinical studies on a range of neuropsychiatric and neurological disorders. This journal is indexed on PubMed Central, the 'PsycINFO' database and CAS, and is the official journal of The International Neuropsychiatric Association (INA). The manuscript management system is completely online and includes a very quick and fair peer-review system, which is all easy to use. Visit http://www.dovepress.com/testimonials.php to read real quotes from published authors.

Submit your manuscript here: http://www.dovepress.com/neuropsychiatric-disease-and-treatment-journal 